\title{
ON A NEW APPROACH TO CONVOLUTION CONSTRUCTIONS
}

\author{
S.B. YAKUBOVICH
}

Department of Mathematics \& Mechanics

Byelorussian State University

P. 0. B0x - 385. Minsk - 50

Byelorussia

and

SHYAM L. KALLA

Research Center for Applied Mathematics

Engineering Faculty

Zulia University

Apartado - 10182

Maracaibo - Venezuela.

(Received October 3, 1991 and in revised form April 7, 1992)

ABSTRACT. In this paper we establish some new approach to constructing convolution for general Mellin type transforms. This method is based on the theory of double Mellin-Barnes integrals. Some properties of convolutions and several examples are given.

KEY WORDS AND PHRASES. Convolution, Mellin-Barnes Integrals G-transforms, 1991 AMS SUBJECT CLASSIFICATION CODES. 44A35, 44A15, 33C40.

1. INTRODUCTION.

As is known from [1], the following operators of Mellin tyve convolution

$$
(k f)(x)=\int_{0}^{\infty} k\left(\frac{x}{u}\right) f(u) \frac{d u}{u}, x>0,
$$

define a sufficiently large class of integral operators. In particular, if $k(x)=e^{-x}$, then formula (1.1) defines the modified Laplace transform $\left(\Lambda_{+} f\right)[2]$

$$
\left.\left(\Lambda_{+} f\right)(x)=\int_{0}^{\infty} e^{-x / u_{f}} f u\right) \frac{d u}{u} .
$$

which leads to classical Laplace transform (Lf) [3] with the following convolution

$$
(f * g)(x)=\int_{0}^{x} f(x-t) g(t) d t
$$

and the factorization equality

$$
(L(f * g))(x)=(L f)(x)(L g)(x) .
$$


Recently we [4] have developed a method of generalization of the Laplace convolution (1.3) on Mellin type transforms (1.1). It can be effectively extended to the integral transforms with G-function in kernel and more general G-transforms [5]. In this case the factorization equality of type (1.4) takes the following form

$$
\left(k_{1}(f * g)\right)(x)=\left(k_{2} f\right)(x)\left(k_{3} g\right)(x),
$$

where $(f * g)(x)$ is general convolution which is defined below for three operators $\left(K_{1}, K_{2}, K_{3}\right)$. In case of classical Laplace transform it is the set $(L, L, L)$, for $G$-transforms it is $\left(G_{1}, G_{2}, G_{3}\right)$ (see below).

In this paper we consider some properties of these convolutions in special spaces and their various integral representations. By this method one can obtain the known convolutions and many new examples.

2. SOME FUNCTIONAL SPACES, G-TRANSFORM AND ITS PARTICULAR CASES

In this section we will consider generalization of the Mellin type convolution transform (1.1) by Mellin-Parseval equality [2]

$$
(k f)(x)=\frac{1}{2 \pi i} \int_{\gamma-i \infty}^{\gamma+i \infty} k^{*}(s) f^{*}(s) x^{-s} d s,
$$

where * denotes the Mellin transform of functions $k(x), f(x)$

$$
f^{*}(s)=\int_{0}^{\infty} f(x) x^{8-1} d x,
$$

$(\gamma-i \infty, \gamma+i \infty)$ is some vertical contour in the complex plane $s$. Thus the transform (1.1) can be studied with aid of asymptotic of function $k^{*}(s)$ and $f^{*}(s)$ on the contour $\sigma=(\gamma-i x, \gamma+i \infty)$, in particular, when $\sigma=\{s, \operatorname{Re}(s)=\gamma=1 / 2\}$. Our main aim is to consider transforms of the form (2.2) which are convenient for our further studies.

Thus the behaviour of the functions $k^{*}(s)$ and $f^{*}(s)$ on the contour $\sigma$ can observed from the fact that their inverse Mellin transforms belong to the space of functions $M^{-1}(L)$ [5]. As is shown below this space is very convenient for the studies of transform (2.2).

DEFINITION 1 . Denote by $m^{-1}(L)$ the space of functions $f(x), x \in$ $(0,+\infty)$, representable by inverse Mellin transform of integrable functions $f^{*}(s) \in L_{1}(\sigma) \equiv L(\sigma)$ on the contour $\sigma$ :

$$
f(x)=f^{-1}\left\{f^{*}(s) ; x\right\}=\frac{1}{2 \pi i} \int_{\sigma}^{*}(s) x^{-s} d s
$$

The space $M^{-1}(L)$ with the usual operations of addition and multiplication by scalar is linear vector space. If the norm in $n^{-1}(L)$ is introduced by the formula

$$
\|f\|_{R^{-1}(t)}=\int_{-\infty}^{+\infty}\left|f^{*}(1 / 2+i t)\right| d t,
$$

then the space $\mathrm{M}^{-1}(L)$ is a Banach space.

Now we consider main properties of the space $m^{-1}(L)$.

1) $f(x) \in \mathbb{R}^{-1}(L)$ if and only if $x^{-1} f\left(x^{-1}\right) \in \mathbb{M}^{-1}(L)$.

This property follows from the fact that the functions $f(x)$ and 
$x^{-1} f\left(x^{-1}\right)$ are the inverse Mellin transforms of the functions $f(s)$ and $f^{*}(1-s)$ respectively, But the functions $f^{*}(s)$ and $f^{*}(1-s)$ either both belong to $L(\sigma)$ or none of them belong to $L(\sigma)$.

2) If $f(x) \in \mathbb{R}^{-1}(L)$ then $x^{1 / 2} f(x)$ is bounded uniformly, continuous on $(0,+\infty)$ and furthermore $x^{1 / 2} f(x)=0(1)$ when $x \rightarrow \infty$ and $x \rightarrow 0$.

This property follows from the Riemann - Lebesgue lemma

3) If $f(x), g(x) \in \mathbb{R}^{-1}(L)$ then $x^{1 / 2} f(x) g(x) \in \mathbb{R}^{-1}(L)$.

This fact follows from that $x^{1 / 2} f(x) g(x)$ is the inverse Mellin transform of the function $\frac{1}{2 \pi i} \int f^{*}(\tau) g^{*}(s-\tau+1 / 2) d \tau$ which belongs to $L(\sigma)$ by Fubini theorem. Here $g(x)=g^{-1}\left\{g^{*}(s) ; x\right\}$.

4) Let $f(x) \in R^{-1}(L)$ and $x^{-1 / 2} g(x) \in L\left(R_{+}\right)$. Then

$$
\int_{0}^{\infty} g(u) f\left(\frac{x}{u}\right) \frac{d u}{u} \text { belongs to } \mathbb{s}^{-1}(L) \text {. }
$$

In fact by the property of the Mellin convolution this integral is the inverse Mellin transform of the. function $f^{*}(s) g^{*}(s)$ and since $f^{*}(s) \in L(\sigma)$ and $g^{*}(s)$ belongs to space of essential bounded functions, hence $f^{*}(s) g^{*}(s) \in L(\sigma)$.

As is known by [1] the Mellin transform of G-function is the ratio of products of gamma-functions and according to asymptotic expansion of gamma-functions this ratio has power-exponential behavior on the contour $\sigma$. Therefore it is necessary to take into consideration this fact in the spaces of, $\mathrm{R}^{-1}(L)$ type.

DEFINITION 2.[5]. Let $c, \gamma \in R$ be such that

$$
2 \operatorname{sgn}(c)+\operatorname{sgn}(\gamma) \geq 0 \text {. }
$$

Denote by $R_{c, \gamma}^{-1}(L)$ the space of functions $f(x), x \in(0,+x)$, representable by the inverse Mellin transform $(2.3)$, where $f^{*}(s)|s|^{\gamma} x$ $x e^{\pi c|I m s|} \in L(\sigma)$.

Note that $\left.f^{*}(s)|s|^{\gamma} e^{\pi_{c}|\operatorname{lms}|}\right|_{\epsilon \mid L(\sigma)} \mid$ if and only if $f^{*}(s)|s|^{\gamma_{e} \pi_{c}|s| \epsilon}$ $L(\sigma)$, and in this case the integral in (2.3) converges if $c>0, \gamma \in R$, or $c=0, \gamma>0$, which is equivalent to (2.5).

The space $R_{C, \gamma}^{-1}(L)$ is Banach space with norm

$$
\|f\|_{R_{C, \gamma}^{-1}(L)}=\int_{\sigma} e^{\pi c|I m s|}\left|s^{\gamma} f^{*}(s)\right| d s
$$

It is obvious that the space ${ }_{C, \gamma}^{-1}(L)$ in case $c=0, \gamma=0$ coincides with the space $\mathrm{M}^{-1}(L)$.

DEFINITION 3. The G-transform of function $f(x), x>0$, is defined as

$$
\text { (Gf) }(x)=G_{p, q}^{m, n}\left(\begin{array}{l}
(\alpha)_{p} \\
(\beta)_{q}
\end{array}\right) 0[f(u)](x)=\frac{1}{2 \pi i} \int \psi(s) f^{*}(s) x^{-s} d s, x>0 \text {, }
$$

where $\sigma=\{s \in \mathbb{C}, \operatorname{Re}(s)=1 / 2\}, 0 \leq m \leq p, 0 \leq n \leq q$,

$$
\psi(s)=\frac{\prod_{j=1}^{m} \Gamma\left(\beta_{j}+s\right) \prod_{j=1}^{n} \Gamma\left(1-\alpha_{j}-s\right)}{\prod_{j=n+1}^{p} \Gamma\left(\alpha_{j}+s\right) \prod_{j=m+1}^{q} \Gamma\left(1-\beta_{j}-s\right)} .
$$


Here, we assume that

$$
\left\{\begin{array}{l}
\operatorname{Re}\left(\beta_{j}\right)+1 / 2>0, j=1, \ldots, m ; 1 / 2-\operatorname{Re}\left(\alpha_{j}\right)>0, j=1, \ldots, n ; \\
\operatorname{Re}\left(\alpha_{j}\right)+1 / 2>0, j=n+1, \ldots, P ; 1 / 2-\operatorname{Re}\left(\beta_{j}\right)>0, j=m+1, \ldots, q
\end{array}\right.
$$

DEFIMITION 4. The ordered pair $\left(c^{*}, \gamma^{*}\right)$, where

$$
c^{*}=m+n-\frac{p+q}{2}, \quad \gamma *=R e\left(\sum_{j=1}^{p} \alpha_{j}-\sum_{j=1}^{q} \beta_{j}\right)
$$

is called the index of the G-transform (2.7)

THEOREM 1.[5]. The G-transform with the index $\left(c^{*}, \gamma^{*}\right)$ exists on the space ${ }_{C, \gamma}^{-1}(L)$ if and only if

$$
2 \operatorname{sgn}\left(c+c^{*}\right)+\operatorname{sgn}\left(\gamma+\gamma^{*}\right) \geq 0 \text {. }
$$

In this case the G-transform maps $m_{C, \gamma}^{-1}(L)$ isomorphically into $i_{c+c}^{-1}, \gamma+\gamma^{*}(L)$.

Now wo seo that the inversion of G-transform (2.7) is also G-transform and it can be written as follows

$$
\begin{gathered}
{\left[G_{p, q}^{m, n}\left(\begin{array}{l}
(\alpha)_{1, p} \\
(\beta)_{1, q}
\end{array}\right]\right]^{-1} \circ[g(u)](x)=G_{q, p}^{p-n, q-m}\left(\begin{array}{l}
(\beta)_{m+1, q} \cdot(\beta)_{1, m} \\
(\alpha)_{n+1, p},(\alpha)_{1, n}
\end{array}\right) \circ[g(u)](x)=} \\
=\frac{1}{2 \pi i} \int_{\sigma} \frac{1}{\psi(s)} g^{*}(s) x^{-s} d s
\end{gathered}
$$

The following theorem represents our G-transform defined by contour integral (2.7) in the traditional real form. The proof is obvious from the Mellin - type convolution (1.1).

THEOREM 2. Let

$$
2 \operatorname{sgn}\left(c^{*}\right)+\operatorname{sgn}\left(\gamma^{*}-1\right)>0 \text {, }
$$

then the G-transform (2.7) with the index $\left(c^{*}, \gamma^{*}\right)$ can be represented in the real form (1.1) as follows

$$
\text { (Gf) }(x)=\int_{0}^{\infty} G_{p, q}^{m, n}\left(\left.\frac{x}{u}\right|_{(\beta)_{q}} ^{(\alpha)_{p}}\right) f(u) \frac{d u}{u} \text {. }
$$

where $G_{p, q}^{m, n}\left(x \mid \begin{array}{l}(\alpha)_{p} \\ (\beta)_{q}\end{array}\right)=\frac{1}{2 \pi i} \int_{\sigma} \psi(s) x^{-s}$ ds is Meijer's G-function [1].

Here the existence of the G-transform (2.7) is guaranteed by (2.13) and inequality (2.5) in Definition 2 of $m_{c, \gamma}^{-1}(L)$ (see also (2.11)). Theorem 2 shows that inequality (2.13) provides the convergence of integral which is used for definition of Meijer's G-function.

REMARK 1. If inequality $(2.13)$ is replaced by

$$
4 \operatorname{sgn}\left(c^{*}\right)+2 \operatorname{sgn}\left(\gamma^{*}\right)+\operatorname{sgn}|p-q|>0 \text {. }
$$

then the statement in Theorem 2 is also true (see [5]).

Further,since the Meijer's G-function is rather a general function, its particular cases lead to a number of corresponding transforms. Here we give a table of those transforms which are required to study 
the convolutions. For convenience we introduce the following notations of transforms (see [2]).

$$
\begin{aligned}
& \{k(x)\} \circ[f(u)]=\int_{0}^{\infty} k\left(\frac{x}{u}\right) f(u) \frac{d u}{u}=\frac{1}{2 \pi i} \int_{L} k^{*}(s) f^{*}(s) x^{-s} d s, \\
& \left\{x^{\alpha} k(x)\right\} \circ[f(u)]=\int_{0}^{\infty}\left(\frac{x}{u}\right)^{\alpha} k\left(\frac{x}{u}\right) f(u) \frac{d u}{u}=x^{\alpha}\{k(x)\} \circ\left[x^{-\alpha} f(x)\right]= \\
& =\left(x^{\alpha} k(x) x^{-\alpha}\right)(f)(x), \\
& \{k(x)\} \circ[f(u)]=\frac{1}{2 \pi i} \int_{L} \frac{1}{k^{*}(s)} f^{*}(s) x^{-s} d s, \\
& \left\{x^{\alpha} k(x)\right\}^{-1}[f(u)]=\frac{1}{2 \pi i} \int_{L} \frac{1}{k^{*}(s+\alpha)} f^{*}(s) x^{-s} d s .
\end{aligned}
$$

Now we give a table of the definitions of important simple G-transforms in the forms (2.15)-(2.18), where the function $\psi(s)$ $\left(k^{*}(s)\right)$ is defined by $(2.8)$ such that $p+q \leq 2$. These transforms are special modifications of known classical integral transforms and their inversions (Laplace transform, Hankel transform, Stieltjes transform, Riemann-Liouville differintegral operators, Meijer transform).

The more general particular cases of G-transforms, which will be introduced in section 4, can be easily obtained from (2.15)-(2.18) by using the table of Mellin transforms and representations of the kernels $k(x)$ through Meijer's G-function [6].

TABLE 1

SIMPLE INTEGRAL G-TRANSFORMS AND THEIR INVERSIONS Modified Laplace transforms

$$
\begin{aligned}
& G_{0,1}^{1,0}\left(\frac{-}{0}\right) \circ[f(u)](x)=\left\{e^{-x}\right\} \circ[f(u)]=\Lambda_{+} f(x)=\int_{0}^{\infty} e^{-(x / u)} f(u) \frac{d u}{u} \quad(2.19) \\
& G_{1,0}^{0,1}\left(\frac{1}{-}\right) \circ[f(u)](x)=\left\{e^{-1 / x}\right\} \circ[f(u)]=\Lambda_{-} f(x)=\int_{0}^{\infty} e^{-(x / u)^{-1}} f(u) \frac{d u}{u}(2.20) \\
& \left\{x^{\alpha} e^{-x^{ \pm 1}}\right\} \circ[f(u)]=x^{\alpha} \Lambda_{ \pm} x^{-\alpha} f(x)=x^{\alpha} \int_{0}^{\infty} e^{-(x / u)^{ \pm 1}} u^{-\alpha} f(u) \frac{d u}{u} \\
& {\left[G_{0,1}^{1,0}\left(\frac{-}{0}\right)\right]^{-1} \circ[f(u)](x)=\left\{e^{-x}\right\}^{-1} \circ[f(u)]=\Lambda_{+}^{-1} f(x)=\frac{1}{2 \pi i} \int \frac{1}{\Gamma(s)} f^{*}(s) x^{-s} d s} \\
& \left\{x^{\alpha} e^{-x^{ \pm 1}}\right\}^{-1} \circ[f(u)]=x^{\alpha} \Lambda_{ \pm}^{-1} x^{-\alpha} f(x)=\frac{1}{2 \pi i} \int_{\Gamma( \pm(s+\alpha))} f^{*}(s) x^{-s} d s
\end{aligned}
$$

Modified Riemann-Liouville differintegral operators and their inversions

$$
\begin{aligned}
& G_{1,1}^{0,1}\left(\begin{array}{l}
\alpha \\
0
\end{array}\right) \circ[f(u)](x)=\left\{\frac{(x-1)_{+}^{\alpha-1}}{\Gamma(\alpha)}\right\} \circ[f(u)]=I_{+}^{\alpha}\left(x^{-\alpha} f(x)\right)= \\
& =\int_{0}^{x} \frac{(x-u)^{\alpha-1}}{\Gamma(\alpha)} u^{-\alpha} f(u) d u, \operatorname{Re}(\alpha)>0
\end{aligned}
$$


440

S.B. YAKUBOVICH AND S.L. GALA

$$
\begin{aligned}
& =\frac{d^{n}}{d x^{n}} \int_{0}^{x} \frac{(x-u)^{\alpha+n-1}}{\Gamma(\alpha+n)} u^{-\alpha} f(u) d u, \quad-n<\operatorname{Re}(\alpha)<-n+1 \text { or } \operatorname{Re}(\alpha)=-n \text {, } \\
& \operatorname{Im}(\alpha) \neq 0, n=1,2,3, \ldots \\
& G_{1,1}^{1,0}\left(\begin{array}{l}
\alpha \\
0
\end{array}\right) \circ[f(u)](x)=\left\{\frac{(1-x)_{+}^{\alpha-1}}{\Gamma(\alpha)}\right\} \circ[f(u)]=I_{-}^{\alpha}\left(x^{-\alpha} f(x)\right)= \\
& =\int_{x}^{\sigma} \frac{(u-x)^{\alpha-1}}{\Gamma(\alpha)} u^{-\alpha} f(u) d u, R \theta(\alpha)>0 \\
& =\left(-\frac{d}{d x}\right)^{n} \int_{x}^{\infty} \frac{(u-x)^{\alpha+n-1}}{\Gamma(\alpha+n)} u^{-\alpha} f(u) d u,-n<\operatorname{Re}(\alpha)<-n+1 \text { or } \operatorname{Re}(\alpha)=-n \text {, } \\
& \operatorname{Im}(\alpha) \neq 0, n=1,2,3, \ldots \\
& \left\{\frac{( \pm 1 \mp x)_{+}^{\alpha-1}}{\Gamma(\alpha)}\right\}^{-1} \circ[f(u)]=\left(I_{ \pm}^{\alpha}\right)^{-1}\left(x^{-\alpha} f(x)\right)=I_{ \pm}^{-\alpha}\left(x^{-\alpha} f(x)\right)
\end{aligned}
$$

Modified Wankel transforms and their inversions

$$
\begin{aligned}
& G_{0,2}^{1,0}(\nu / 2,-\nu / 2) \circ[f(u)](x)=\left\{J_{\nu}(2 \sqrt{x})\right\} \circ[f(u)]=\int_{0}^{\infty} J_{\nu}(2 \sqrt{x / u}) f(u) \frac{d u}{u}(2.27) \\
& G_{2,0}^{0,1}\left({ }^{1-\nu / 2,1+\nu / 2}\right) \circ[f(u)](x)=\left\{J_{\nu}\left(\frac{2}{\sqrt{x}}\right)\right\} 0[f(u)]=\int_{0}^{\infty} J_{\nu}\left(2 \sqrt{u / x} f(u) \frac{d u}{u}(2.28)\right. \\
& {\left[G_{0,2}^{1,0}(\nu / 2,-v / 2)\right]^{-1} \circ[f(u)](x)=\left\{J_{\nu}(2 \sqrt{x})\right\}^{-1} \circ[f(u)]=} \\
& =\left\{x^{-1} J_{v}\left(\frac{2}{\sqrt{x}}\right)\right\} 0[f(u)] \\
& {\left[G_{2,0}^{0,1}(1-v / 2,1+\nu / 2)\right]^{-1} \circ[f(u)](x)=\left\{J_{\nu}\left(\frac{2}{\sqrt{x}}\right)\right\}^{-1} \circ[f(u)]=} \\
& =\left\{x J_{\nu}(2 \sqrt{x})\right\} \circ[f(u)]
\end{aligned}
$$

Modified Sin- and Cos - transforms and their inversions

$$
\begin{gathered}
G_{0,2}^{1,0}(1 / 2,0) \circ[f(u)](x)=\left\{\frac{1}{\sqrt{\pi}} \sin (2 \sqrt{x})\right\} \circ[f(u)]=\frac{1}{\sqrt{\pi}} \int_{0}^{\infty} \sin (2 \sqrt{x / u}) f(u) \frac{d u}{u} \\
G_{0,2}^{1,0}(0,1 / 2) \circ[f(u)](x)=\left\{\frac{1}{\sqrt{\pi}} \cos (2 \sqrt{x})\right\}_{0}[f(u)]=\frac{1}{\sqrt{\pi}} \int_{0}^{\infty} \cos (2 \sqrt{x / u}) f(u) \frac{d u}{u}
\end{gathered}
$$


NEW APPROACH TO CONVOLUTION CONSTRUCTIONS

441

$$
\begin{gathered}
=\frac{1}{\sqrt{\pi}} \int_{0}^{u} \sin (2 \sqrt{u / x}) f(u) \frac{d u}{u} \\
G_{2,0}^{0,1}\left(\frac{1 / 2,1 / 2}{-}\right)[f(u)](x)=\left\{\frac{1}{\sqrt{\pi}} \cos \left(2 x^{-1 / 2}\right)\right\} \circ[f(u)]= \\
=\frac{1}{\sqrt{\pi}} \int_{0}^{\infty} \cos (2 \sqrt{u / x}) f(u) \frac{d u}{u} \\
{\left[G_{2,0}^{0,1}(1 / 2,0)\right]^{-1} \circ[f(u)](x)=\left\{\frac{1}{\sqrt{\pi}} \sin (2 \sqrt{x})\right\}^{-1} \circ[f(u)]=} \\
=\frac{1}{\sqrt{\pi}}\left\{x^{-1 / 2} \sin \left(2 x^{-1 / 2}\right)\right\} \circ[f(u)] \\
{\left[G_{2,0}^{0,1}(0,1 / 2)\right]^{-1} \circ[f(u)](x)=\left\{\frac{1}{\sqrt{\pi}} \cos (2 \sqrt{x})\right\}^{-1} \circ[f(u)]=} \\
=\frac{1}{\sqrt{\pi}}\left\{x^{-1 / 2} \cos \left(2 x^{-1 / 2}\right)\right\} \circ[f(u)] \\
=\frac{1}{\sqrt{\pi}}\left\{x^{1 / 2} \sin (2 \sqrt{x})\right\} \circ[f(u)] \\
{\left[G_{2,0}^{0,1}\left[-\frac{1 / 2,1}{\sqrt{\pi}} \sin \left(2 x^{-1 / 2}\right)\right\}^{-1} \circ[f(u)](x)=\{f(u)]=\right.} \\
=\frac{1}{\sqrt{\pi}}\left\{x^{1 / 2} \cos (2 \sqrt{x})\right\} \circ[f(u)]
\end{gathered}
$$

Modified stieltjes transform and its inversion

$$
\begin{aligned}
& G_{1,1}^{1,1}\left(\begin{array}{c}
1-\rho \\
0
\end{array}\right) \circ[f(u)](x)=\left\{\Gamma(\rho)(1+x)^{-\rho}\right\} \circ[f(u)]= \\
&= \Gamma(\rho) \int_{0}^{\infty} \frac{u^{\rho} f(u)}{(x+u)^{\rho}} \frac{d u}{u} \\
& {\left[G_{1,1}^{1,1}\left(\begin{array}{c}
1-\rho \\
0
\end{array}\right)\right]^{-1} \circ[f(u)] }(x)=\left\{\Gamma(\rho)(1+x)^{-\rho}\right\}^{-1} \circ[f(u)]= \\
& \frac{1}{2 \pi i} \int_{L} \frac{1}{\Gamma(s) \Gamma(\rho-s)} f^{*}(s) x^{-s} d s
\end{aligned}
$$

Modified Meijer transforms and their inversions

$$
\mathrm{G}_{0,2}^{2,0}(\nu / 2,-\nu / 2) \cdot[f(u)](x)=\left\{2 k_{\nu}(2 \sqrt{x})\right\} \circ[f(u)]=
$$




$$
\begin{aligned}
& =2 \int_{0}^{\infty} k_{\nu}\left(2 \sqrt{x / u} f(u) \frac{d u}{u}\right. \\
& G_{2,0}^{0,2}\left(\begin{array}{c}
1-\nu / 2,1+\nu / 2 \\
-
\end{array}\right) \cdot[f(u)](x)=\left\{2 K_{\nu}\left(\frac{2}{\sqrt{x}}\right)\right\} \circ[f(u)]=2 \int_{0}^{\infty} K_{v}(2 \sqrt{u / x}) f(u) \frac{d u}{u} \\
& {\left[G_{0,2}^{2,0}(\nu / 2,-\nu / 2)\right]^{-1} \circ[f(u)](x)=\left\{2 K_{\nu}(2 \sqrt{x})\right\}^{-1} \circ[f(u)]=} \\
& =\frac{1}{2 \pi i} \int_{L} \frac{1}{\Gamma(s+\nu / 2) \Gamma(s-\nu / 2)} f^{*}(s) x^{-s} d s \\
& {\left[G_{2,0}^{0,2}\left(\begin{array}{c}
1-\nu / 2,1+\nu / 2 \\
-
\end{array}\right]\right]^{-1} \circ[f(u)](x)=\left\{2 K_{\nu}\left(\frac{2}{\sqrt{x}}\right)\right\}^{-1} \circ[f(u)]=} \\
& =\frac{1}{2 \pi i} \int_{L} \frac{1}{\Gamma(s+\nu / 2) \Gamma(-s-\nu / 2)} f^{*}(s) x^{-s} d s
\end{aligned}
$$

3. DEFINITION AND MAIN PROPERTIES OF CONVOLUTIONS FOR MELLIN TYPE TRANSFORMS

Now we consider the Laplace convolution (1.3) if $f(x), g(x) \epsilon$ $m^{-1}(L)$. By Definition 1 substituting representations (2.3) for $f(x)$, $g(x)$ in (1.3), changing order of integration and using the beta-integral we can represent $(1.3)$ in its equivalent form:

$$
\left(f_{*}^{L} g\right)(x)=\frac{x}{(2 \pi i)^{2}} \int_{\sigma_{t}} \int_{s} \frac{\Gamma(1-s) \Gamma(1-t)}{\Gamma(2-s-t)} f^{*}(s) g^{*}(t) x^{-8-t} d s d t \text {, }
$$

where $\sigma_{8} \times \sigma_{t}=\left\{(s, t) \in \mathbb{C}^{2}, \operatorname{Re}(s)=\operatorname{Re}(t)=1 / 2\right\}$.

For Laplace convolution (3.1) we have the following result.

THEOREM 3. The classical Laplace convolution $\left(f_{*}^{L} g ;(x)(1.3)\right.$ exists for all $f(x), g(x) \in M^{-1}(L)$ and it posseses the factorization property (1.4). In this case $x^{-1 / 2}\left(f_{*}^{L} g\right)(x) \in x^{-1}(L)$.

PROOF. From (3.1) it follows that the Laplace convolution $\left(f_{*}^{L} g\right)(x)$ exists if and only if

$$
\frac{\Gamma(1-s) \Gamma(1-t)}{\Gamma(2-s-t)} f^{*}(s) g^{*}(t) \in L\left(\sigma_{s} \times \sigma_{t}\right)
$$

Using the representation for the beta-function we obtain that

$$
\frac{\Gamma(1-s) \Gamma(1-t)}{\Gamma(2-s-t)}=O(1) \text {, when } s, t \rightarrow \infty,(s, t) \in \sigma_{s} \times \sigma_{t} \text {. }
$$

Bit by Definition 1 it follows that $f^{*}(s) g^{*}(t) \epsilon \quad L\left(\sigma_{s} \times \sigma_{t}\right)$. Hence the condition (3.2) holds valid. Further by the substitution $\tau=s+t-1 / 2$ representation (3.1) can be written in the following form:

$$
\left(f_{*}^{L} g\right)(x)=\frac{x^{1 / 2}}{2 \pi i} \int_{\sigma} \frac{x^{-\tau}}{\Gamma(3 / 2-\tau)} d \tau \frac{1}{2 \pi i} \int_{\sigma_{t}} \Gamma(1 / 2-\tau+t) \Gamma(1-t) f^{*}(\tau-t+1 / 2) g^{*}(t) d t \text {, }
$$

where $\sigma_{\tau}=\{\tau \in \mathbb{C}, \operatorname{Re}(\tau)=1 / 2\}$. 
Hence it follows that $x^{-1 / 2}\left(f_{*}^{L} g\right)(x) \in M^{-1}(L)$. Now by an appeal to the classical Laplace transform

$$
(L f)(x)=\int_{0}^{x} e^{-x u_{f}} f(u) d u
$$

and the convolution (3.1) it is not difficult to obtain the property (1.4) Theorem 3 is proved.

The representation (3.1) for Laplace convolution can be modified and generalized on the G-transform (2.7).

DEFINITION 5. Integral convolution $(f * g)(x)$ for two functions $f(x)$ and $g(x)$ for the set of three $G$-transforms $\left(G_{1}, G_{2}, G_{3}\right)$ is defined as the following integral.

$$
(f * g)(x)=\frac{1}{(2 \pi i)^{2}} \iint_{\sigma_{s} \sigma_{t}} \frac{H_{2}(s) H_{3}(t)}{H_{1}(s+t)} f^{*}(s) g^{*}(t) x^{-s-t} d s d t, x>0 \text {, }
$$

where $H_{j}(s), j=1,2,3$ are the corresponding kernels of G-transforms $(G, f)(x)$ which are defined by the following relations:

$H_{j}(s)=\Gamma\left[\begin{array}{l}\left(\beta_{m_{j}^{j}}^{j}\right)+s, 1-\left(\alpha_{n j}^{j}\right)-s \\ \left(\alpha_{p_{j}^{j},{ }_{j}+1}\right)+s, 1-\left(\beta_{q_{j}, m_{j}+1}\right)-s\end{array}\right]=\frac{\prod_{k=1}^{m_{j}} \Gamma\left(\beta_{k}^{j}+s\right) \prod_{k=1}^{n} \Gamma\left(1-\alpha_{k}^{j}-s\right)}{\prod_{k=n_{j}+1}^{p_{j}} \Gamma\left(\alpha_{k}^{j}+s\right) \prod_{k=m, 1}^{q} \Gamma\left(1-\beta_{k}^{j}-s\right)}$ (3.6)

Thus by Definition 5 we see that for each convolution defined by (3.5) there corresponds the ordered set $\left(G_{1}, G_{2}, G_{3}\right)$ of $G$-transforms and $v i c e$ versa. Hence we obtain the family of integral convolutions for corresponding G-transforms. As is known from [5], the composition of G-transform is also G-transform. Hence we can note some algebraic and structural properties of these convolutions (see below).

THEOREM 4. Let $f(x), g(x) \in \mathbb{R}_{L, \gamma}^{-1}(L)$ and for parameters of the kerriels $H_{j}(s), j=1,2,3$ and $H_{1}(s+1 / 2)$ the corresponding conditions (2.9) hold. Let further,

$$
(s, t) \in \sup _{s} \times \sigma_{t}\left[|\psi(s, t)| \exp (-\pi c(|s|+|t|))|s t|^{-\gamma}\right]<t, \infty .
$$

where $\psi(s, t)=\left[H_{1}(s+t)\right]^{-1} H_{2}(s) H_{3}(t)$. Then the convolution (3.5) exists and $x^{1 / 2}(f * g)(x) \in N^{-1}(L)$.

PROOF. By condition (3.7) and Definition 2 it follows that $\psi(s, t) f^{*}(s) g^{*}(t) \in L\left(\sigma_{s} \times \sigma_{t}\right)$. Hence we write the integral (3.5) in the following form (see 3.4 ).

$(f * g)(x) \frac{x^{-1 / 2}}{2 \pi i} \int_{\sigma_{\tau}} \frac{x^{-\tau} d \tau}{H_{1}(\tau+1 / 2)} \frac{1}{2 \pi i} \int_{\sigma_{t}} H_{2}(1 / 2+\tau-t) f^{*}(1 / 2+\tau-t) H_{3}(t) g^{*}(t) d t$

and using Definition 1 the proof of Theorem 4 is completed.

THEOREM 5. Let the conditions of Theorem 4 hold and moreover the following inequalities hold

$$
2 \operatorname{sgn}\left(c+c_{j}^{*}\right)+\operatorname{sgn}\left(\gamma+\gamma_{j}^{*}\right) \geq 0, j=2,3 \text {, }
$$


where $c_{1}^{*}, \gamma_{1}^{*}, \quad i=2,3-$ indices of G-transforms with corresponding kernels (3.6). Then the factorization equality of type (1.5) for the $G$-transforms set $\left(G_{1}, G_{2}, G_{3}\right)$ is true

$$
\left(G_{1}(f * g)\right)(x)=\left(G_{2} f\right)(x)\left(G_{3} g\right)(x) \text {. }
$$

where

$(G f)(x)=x^{-1 / 2} \hat{G}\left(t^{1 / 2} f\right)(x)$ $\begin{array}{llllll}\text { in case } x^{1 / 2} f(x) & f & { }^{-1}, \gamma(L), & \text { and } G \text {-transform } \hat{G} \text { has the } \\ \text { kernel } \hat{H}(s)=H(s+1 / 2) \text {. } & & & \end{array}$

PROOF. By the property $(3)$ of ${ }^{-1}(L)$ and Mellin-parseval equality (2.1) we notice that the function $h(x)=x^{1 / 2}\left(G_{2} f\right)(x)\left(G_{3} g\right)(x)$ is the inverse Mellin transform of the t-integral of (3.8). Further the conditions of Theorem 5 provide the existence of G-transforms $G, j=2,3$.

Now we apply $G_{1}$-transform to convolution (3.5) and obtain the corresponding factorization equality.

THEOREM 6. Let $\psi(s, t) \in L\left(\sigma_{s} \times \sigma_{t}\right)$ and $f(x), g(x) \in L\left(x^{-1 / 2}, R_{+}\right)$

$\left(x^{-1 / 2} f(x), x^{-1 / 2} g(x) \in L\left(R_{+}\right)\right)$.

Then the following real representation of convolution (3.5) holds:

where

$$
(f * g)(x)=\int_{R_{+}} s\left(\frac{x}{u}, \frac{x}{v}\right) f(u) g(v) \frac{d u d v}{u v} \text {, }
$$

$$
s(x, y)=\iint_{\sigma} \int_{\sigma_{t}} \psi(s, t) x^{-s} y^{-1} d s d t .
$$

The proof of Theorem 6 can be obtained by Fubini theorem.

Now we consider the convolutions in form (3.9), where the corresponding kernels $s(x, y)$ depend on the sum and maximum of variables $x, y$. As it is known from [7] $\psi(s, t)=k^{*}(s+t) B(s, t)$ (for sum) and $\psi(s, t)=(s+t) k^{*}(s+t) s^{-1} t^{-1}$ (for maximum). In the first case $H_{1}(s)=\frac{\Gamma(s)}{k^{*}(s)}$ $H_{2}(s)=H_{3}(s)=\Gamma(s)$ and in the second case $H_{1}(s)=\frac{1}{s K^{*}(s)} \cdot H_{2}(s)=H_{3}(s)=s^{-1}$. where $k^{*}(s)$ is Mellin transform of some function $k(x)$ such that $h(x)=x^{-1 / 2} k(x)$ belongs to the space $R$ [8] of integrable functions on arbitrary segment $[\epsilon, E], 0<\epsilon<E<\infty$. Further the integral

$$
h^{*}(s)=\int_{0}^{x} h(x) x^{s-1} d x, s \in \sigma,
$$

converges, i.e.there exists a constant $C>0$, for which for all $\epsilon$, E>0 and $t \in R$

$$
\left|\int_{\epsilon}^{E} k(x) x^{1 t-1 / 2} d x\right|<c .
$$

REMARK 2. In particular, if $k(x) \in L(0, \infty)$, then $h(x)=x^{1 / 2} k(x) \in R$. 
THEOREM 7. Let $f(x), g(x) \in \mathbb{R}^{-1}, \gamma(L), x^{1 / 2} k(x) \in R$. Then the following integral representations of type (3.9) for convolutions(3.5) (f*g) $(x)$ and $(f * g)_{\max }(x)$ are true

$$
\begin{aligned}
& (f * g)_{+}(x)=\int_{R^{2}} k\left(x \frac{u+v}{u v}\right) f(u) g(v) \frac{d u d v}{u v} . \\
& (f * g)_{\max }(x)=\int_{R_{+}^{2}} k\left(x \max \left\{u^{-1}, v^{-1}\right\}\right) f(u) g(v) \frac{d u d v}{u v} .
\end{aligned}
$$

The proof of Theorem 7 can be obtained by fubini theorem and using of the following identities [7]

$$
\begin{aligned}
& B(s, t) k^{*}(s+t)=\int_{R^{2}} k(x+y) x^{s-1} y^{t-1} d x d y, \\
& \frac{s+t}{s t} k^{*}(s+t)=\int_{R_{+}^{2}} k\left(\max \{x, y\} x^{s-1} y^{t-1} d x d y .\right.
\end{aligned}
$$

By Theorem 3 it is not difficult to obtain the factorization equalities for convolutions $(3.13),(3.14)$.

$$
\begin{aligned}
& \left(G_{+}(f * g)_{+}\right)(x)=\left(\Lambda_{+} f\right)(x)\left(\Lambda_{+} g\right)(x), \\
& \left(G_{\max }(f * g)(x)=\left(I_{-}^{1} x^{-1}\right)(f)(x)\left(I_{-}^{1} x^{-1}\right)(g)(x),\right.
\end{aligned}
$$

where G-transform $G_{+}$and $G_{\max }$ have the kernels as indicated above and operators in right parts of (3.17), (3.18) are defined by Table 1.

Now we consider some estimations of norms for convolutions (3.13), (3.14) in traditional L-spaces.

THEOREM 8. Let $k(x) \in L(0, \infty)$ and $f(x), g(x) \in L\left(x^{-1 / 2} ; 0, \infty\right) .\left(x^{-1 / 2} g(x)\right.$, $L$ $(0, \infty))$. Then the convolutions $(f * g)_{+}(x),(f * g)_{\max }(x) \in L(0, v)$ and the following estimations of norms are true

$$
\left\|(F * g)_{\max }\right\|_{L\left(R_{+}\right)} \leq 2\left\|(f * g)_{+}\right\|_{L\left(R_{+}\right)} \leq M\left\|_{L\left(x^{-1 / 2} ; R_{+} y\right.}\right\| g \|_{L\left(x^{-1 / 2} ; R_{+}\right),}
$$

where $M=\int_{0}^{\infty}|k(x)| d x$.

The proof of Theorem 8 can be obtained by an appeal to the following chain of inequalities and equalities.

$$
\begin{gathered}
\int\left|\int_{\substack{R_{+} \\
R_{+}}} k\left(x \max \left\{u^{-1}, v^{-1}\right\}\right) f(u) g(v) \frac{d u d v}{u v}\right| d x \leq \\
\leq \int_{R_{+}}|k(x)| d x \int_{R_{+}} \min \{u, v\}|f(u) g(v)| \frac{d u d v}{u v}= \\
=\int_{R_{+}}|k(x)| d x \int_{R_{+}} \frac{2 u v}{2 \max \{u, v\}}|f(u) g(v)| \frac{d u d v}{u v} \leq 2 \int_{R_{+}}|k(x)| d x \int_{R_{+}} \frac{u v}{u+v}|f(u) g(v)|
\end{gathered}
$$


$\frac{d u d v}{u v} \leq \int_{R_{+}}|k(x)| d x \int_{R_{+}} \frac{|f(u) g(v)|}{\sqrt{u v}} d u d v \leq \int_{R_{+}}|k(x)| d x \int_{R_{+}} \frac{|f(u)|}{\sqrt{u}} d u \int_{R_{+}} \frac{|g(v)|}{\sqrt{v}} d v$.

EXAMPLE 1. Let $k(x)=2 K_{0}(2 \sqrt{x})$ be MacDonald function [1]. Then the corresponding convolutions (3.13), (3.14) and their factorization equalities can be written in the forms

$$
\begin{gathered}
(f * g)(x)=2 \int_{R_{+}} K_{0}\left(2 \sqrt{x \frac{u+v}{u v}}\right) f(u) g(v) \frac{d u d v}{u v}, \\
\left(\Lambda_{+}^{-1}(f * g)_{+}\right)(x)=\left(\Lambda_{+} f\right)(x)\left(\Lambda_{+} g\right)(x) \\
\left.(f * g)_{\max }(x)=2 \int_{R_{+}} K_{0}\left(2 \sqrt{x \max \left\{u^{-1}, v^{-1}\right.}\right\}\right) f(u) g(v) \frac{d u d v}{u v} \\
\left\{2 x^{1 / 2} K_{1}(2 \sqrt{x})\right\}^{-1} \circ\left[\left(f^{*} g\right)_{\max }(u)\right]=\left(I_{-}^{1} x^{-1}\right)(f)(x)\left(I_{-}^{1} x^{-1}\right)(g)(x) .
\end{gathered}
$$

4. SOME EXAMPLES OF CONVOLUTIONS AND FACTORIZATION EQUALITIES In this section we give a table of examples of integral convolution by real representation (3.9) with $s(x, y)$ as hypergeometric function of two variables (Horn functions)[9]. Some other Horns functions can be defined by the use of G-function of two variables [4]. The sets of G-transforms in factorization equalities follow from Table 1 and table of Mellin transforms in [1], [6].

TABLE 2

$$
\begin{aligned}
(f * g)(x) & =\int_{0}^{\infty} \int_{0}^{\infty} F_{1}\left(\alpha, \beta, \beta^{\prime}, \gamma ;-\frac{x}{u},-\frac{x}{v}\right) f(u) g(v) \frac{d u d v}{u v}, \\
\left(x^{1-\alpha} I_{+}^{\alpha-\gamma} x^{\gamma-1}\right)(f * g)(x)=\frac{\Gamma(\gamma)}{\Gamma(\alpha)}\left\{(1+x)^{-\beta}\right\}(f)(x)\left\{(1+x)^{-\beta^{\prime}}\right\}(g)(x) . & (4,1) \\
(f * g)(x) & =\int_{0}^{\infty} \int_{0}^{\infty} F_{3}\left(\alpha, \alpha^{\prime}, \beta, \beta^{\prime}, \gamma ;-\frac{x}{u},-\frac{x}{v}\right) f(u) g(v) \frac{d u d v}{u v}, \\
\left(x^{-\gamma} \Lambda_{-} x^{\gamma}\right)\left(f^{*} g\right)(x) & =\Gamma(\gamma)\left\{x^{(1-\alpha-\beta) / 2} e^{(2 x)^{-1} w} \frac{1-\alpha-\beta}{2}, \frac{\alpha-\beta}{2}\left(\frac{1}{x}\right)\right\}(f)(x) x \\
x\left\{x^{\left(1-\alpha^{\prime}-\beta^{\prime}\right) / 2} e^{(2 x)}{ }^{-1} w\right. & \left.1-\alpha^{\prime}-\beta^{\prime}, \frac{\alpha^{\prime}-\beta^{\prime}}{2}\left(\frac{1}{x}\right)\right\}(g)(x) . \\
(f * g)(x)= & \int_{0}^{\infty} \int_{0}^{\infty} F_{2}\left(\alpha, \beta, \beta^{\prime}, \gamma, \gamma^{\prime} ;-\frac{x}{u},-\frac{x}{v}\right) f(u) g(v) \frac{d u d v}{u v}, \\
\left(x^{-\alpha} \Lambda_{-}^{-1} x^{\alpha}\right)(f * g)(x) & =\frac{1}{\Gamma(\alpha)}\left\{F_{1}(\beta ; \gamma ;-x)\right\}(f)(x)\left\{F_{1}\left(\beta^{\prime} ; \gamma^{\prime} ;-x\right)\right\}(g)(x) .(4,3)
\end{aligned}
$$




$$
\begin{aligned}
& (f * g)(x)=\int_{0} \int_{1} F_{4}\left(\alpha, \beta, \gamma, \gamma^{\prime} ;-\frac{x}{u},-\frac{x}{v}\right) f(u) g(v) \frac{d u d v}{u v} \text {, } \\
& \frac{1}{2}\left(x^{-(\alpha+\beta) / 2}\left\{k_{\alpha-\beta}\left(\frac{2}{x^{1 / 2}}\right)\right\}^{-1} x^{(\alpha+\beta) / 2}\right)(f * g)(x)=\frac{\Gamma(\gamma) \Gamma\left(\gamma^{\prime}\right)}{\Gamma(\alpha) \Gamma(\beta)} \times \\
& x\left(x^{(1-\gamma) / 2}\left\{J_{\gamma-1}\left(2 x^{1 / 2}\right)\right\} x^{(\gamma-1) / 2}\right\}(f)(x)\left(x^{\left(1-\gamma^{\prime}\right) / 2} x\right. \\
& \left.x\left\{J_{\gamma^{\prime}-1}\left(2 x^{1 / 2}\right)\right\} x^{\left(\gamma^{\prime}-1\right) / 2}\right)(g)(x) \text {. } \\
& (f * g)(x)=\int_{0}^{\infty} \int_{0}^{\infty} \Phi_{1}\left(\alpha, \beta, \gamma ;-\frac{x}{u},-\frac{x}{v}\right) f(u) g(v) \frac{d u d v}{u v} \text {, } \\
& \left(x^{1-\alpha} I_{+}^{\alpha-\gamma} x^{\gamma-1}\right)(f * g)(x)=\frac{\Gamma(\gamma)}{\Gamma(\alpha)}\left\{(1+x)^{-\beta}\right\}(f)(x) \Lambda_{+}(g)(x) \text {. } \\
& (f * g)(x)=\int_{0}^{\infty} \int_{0}^{\infty} \phi_{2}\left(\beta, \beta^{\prime}, \gamma ;-\frac{x}{u},-\frac{x}{v}\right) f(u) g(v) \frac{d u d v}{u v} \text {. } \\
& \left(x^{-\gamma} \Lambda_{-} x^{\gamma}\right)(f * g)(x)=\Gamma(\gamma)\left\{(1+x)^{-\beta}\right\}(f)(x)\left\{(1+x)^{-\beta^{\prime}}\right\}(g)(x) \\
& (f * g)(x)=\int_{0}^{\infty} \int_{0}^{\infty} \phi_{3}\left(\beta, \gamma ;-\frac{x}{u},-\frac{x}{v}\right) f(u) g(v) \frac{d u d v}{u v} \text {, } \\
& \left(x^{-\gamma} \Lambda_{-} x^{\gamma}\right)(f * g)(x)=\Gamma(\gamma)\left\{(1+x)^{-\beta}\right\}(f)(x) \Lambda_{+}(g)(x) \\
& (f * g)(x)=\int_{0}^{\infty} \int_{0}^{\infty} \psi_{1}\left(\alpha, \beta, \gamma, \gamma^{\prime} ;-\frac{x}{u},-\frac{x}{v}\right) f(u) g(v) \frac{d u d v}{u v} \text {. } \\
& \left(x^{-\alpha} \Lambda_{-}^{-1} x^{\alpha}\right)(f * g)(x)=\frac{\Gamma\left(\gamma^{\prime}\right)}{\Gamma(\alpha)}\left\{{ }_{1} F_{1}(\beta ; \gamma ;-x)\right\}(f)(x)\left(x^{\left(1-\gamma^{\prime}\right) / 2} x\right. \\
& \times\left\{J_{\gamma^{\prime}-1}\left(2 x^{1 / 2}\right)\right\} x^{\left(\gamma^{\prime}-1\right) / 2}(g)(x) \\
& (f * g)(x)=\int_{0}^{\infty} \int_{0}^{\infty} \psi_{2}\left(\alpha, \gamma, \gamma^{\prime} ;-\frac{x}{u},-\frac{x}{v}\right) f(u) g(v) \frac{d u d v}{u v} \text {, } \\
& \left(x^{-\alpha} \Lambda_{-}^{-1} x^{\alpha}\right)(f * g)(x)=\frac{\Gamma(\gamma) \Gamma\left(\gamma^{\prime}\right)}{\Gamma(x)}\left(x^{(1-\gamma) / 2} x\right.
\end{aligned}
$$




$$
\begin{aligned}
& \left.x\left\{J_{y-1}\left(2 x^{1 / 2}\right)\right\} x^{(\gamma-1) / 2}\right)(f)(x)\left(x^{\left(1-\gamma^{\prime}\right) / 2} x\right. \\
& \left.x\left\{J_{y^{\prime}-1}\left(2 x^{1 / 2}\right)\right\} x^{\left(\gamma^{\prime}-1\right) / 2}\right)(g)(x) \text {. } \\
& (f * g)(x)=\int_{0}^{\infty} \int_{0}^{\infty} \Xi_{1}\left(\alpha, \alpha^{\prime}, \beta, \gamma ;-\frac{x}{u},-\frac{x}{v}\right) f(u) g(v) \frac{d u d v}{u v} \\
& \left(x^{-\gamma} \Lambda_{-} x^{\gamma}\right)(f * g)(x)=\Gamma(\gamma) \frac{\Gamma\left(1 / 2+\frac{\alpha-\beta}{2}\right) \Gamma\left(\frac{1+\beta-\alpha}{2}\right)}{\Gamma(\alpha) \Gamma(\beta)} \times \\
& x\left\{e^{(2 x)^{-1}} w_{0, \frac{\beta-\alpha}{2}}\left(\frac{1}{x}\right)\right\}(f)(x)\left\{(1+x)^{-\alpha^{\prime}}\right\}(g)(x) . \\
& (f * g)(x)=\int_{0}^{\infty} \int_{0}^{\infty} \Xi_{2}\left(\alpha, \beta, \gamma ;-\frac{x}{u},-\frac{x}{v}\right) f(u) g(v) \frac{d u d v}{u v} \text {. } \\
& \left(x^{-\gamma} \Lambda_{-} x^{\gamma}\right)(f * g)(x)=\Gamma(\gamma) \frac{\Gamma\left(\frac{1+\alpha-\beta}{2}\right) \Gamma\left(\frac{1+\beta-\alpha}{2}\right)}{\Gamma(\alpha) \Gamma(\beta)} \times \\
& x\left\{e^{(2 x)^{-1}} w_{0, \frac{\beta-\alpha}{2}}\left(\frac{1}{x}\right)\right\}(f)(x) \Lambda_{+}(g)(x) .
\end{aligned}
$$

ACKMOWLEDGEMENT. This work was partially funded by a grant from the CONDES of the Zulia university.

\section{REFERENCES}

1.- MARICHEV, O.I.:" Handbook of Integral Iransforms of Higher Iranscendental Functions", Ellis Horwood, Chichester, 1983.

2.- BRYCHKOV, YU.A.; GLAESKE, H-YU., MARICHEV, O.I.: Itogi Nauki i Tekhniki: Mat. Anal., vol. 21, VINITI, 1983, pp. 3-41.; English transl. in J.Soviet Math. 30 (1985), no. 3.

3.- DITKIN, V.A., PRUDNIKOV, A.P.: Itogi Nauki i Tekhniki: Mat. Anal.. VINITI, 1967, PD. 7-82.; English transl. in Progress in Math.vol. 4, Plenum Press, New York, 1969.

4.- YAKUBOVICH, S.B.: DOKI. AN BSSR, 34, N 7, 1990, 588-591.(Russian).

5.- VU KIM TUAN, MARICHEV, O.I.; YAKUBOVICH, S.B.: DOKL. AN SSSR 286. 1986, 786-790; English transl. in Soviet Math. Dokl. 33 (1986).

6.- PRUDNIKOV, A.P.; BRYCHKOV, YU.A.; MARICHEV, O.I.:" Integrals and Series More Special Functions", Gordon and Breach, New York, 1989.

7.- MGUYEN THANH HAI, YAKUBOVICH, S.B.: DOKL. AN BSSR, 34, N 5, 1990, 396-398. (Russian).

8.- VU KIM TUAN: DOK1. AN SSSR, 286, 1986, 521-524; English transl.in Soviet Math. Dokl. 33 (1986).

9.- BATEMAN, H., ERDELYI, A.: Higher Transcendental Functions, McGraw-Hill New York, 1953. 


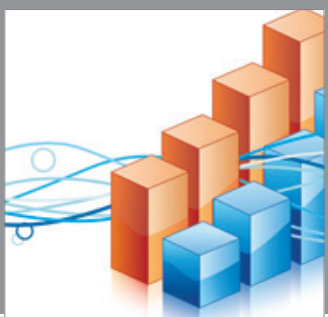

Advances in

Operations Research

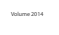

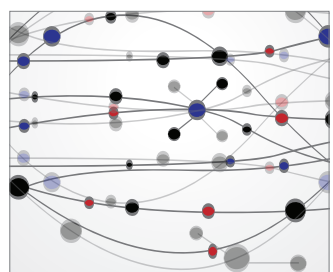

\section{The Scientific} World Journal
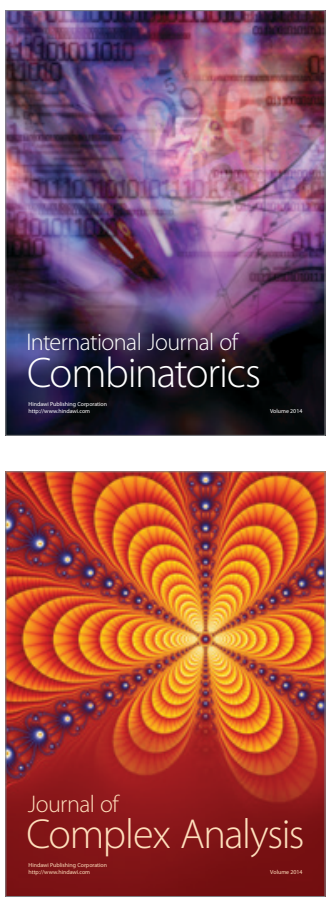

International Journal of

Mathematics and

Mathematical

Sciences
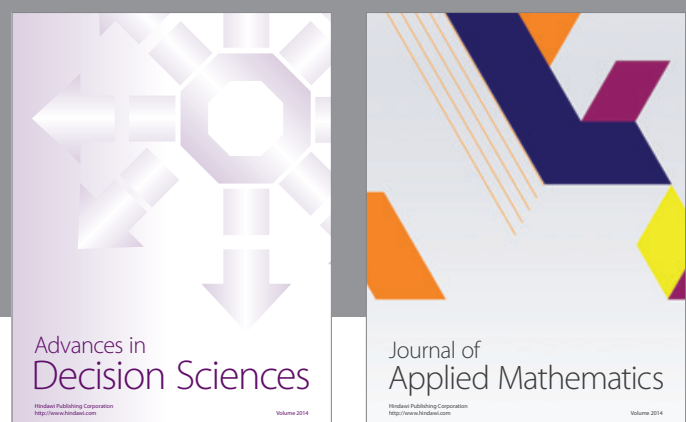

Journal of

Applied Mathematics
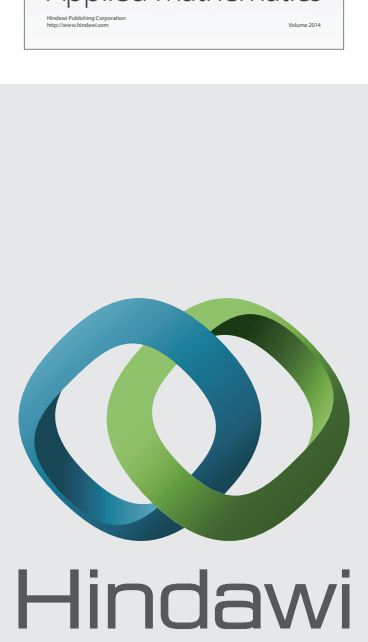

Submit your manuscripts at http://www.hindawi.com
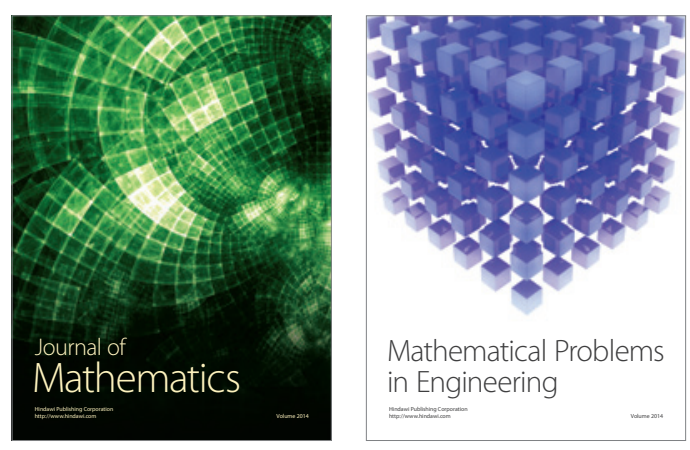

Mathematical Problems in Engineering
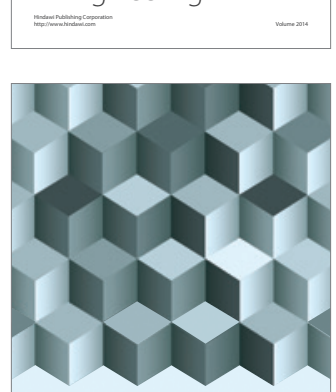

Journal of

Function Spaces
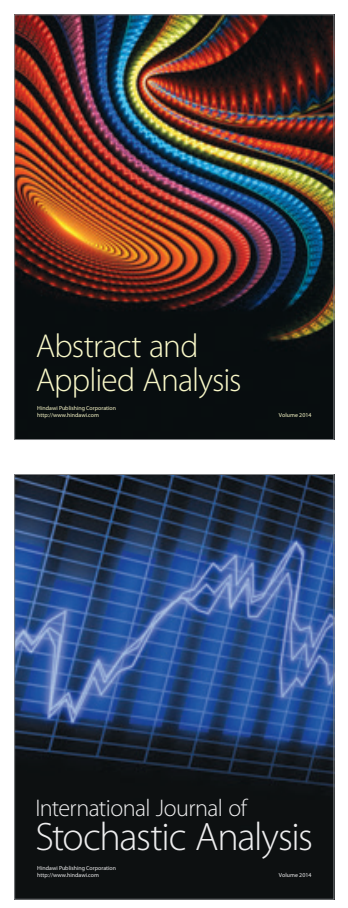

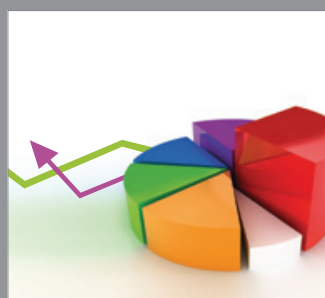

ournal of

Probability and Statistics

Promensencen
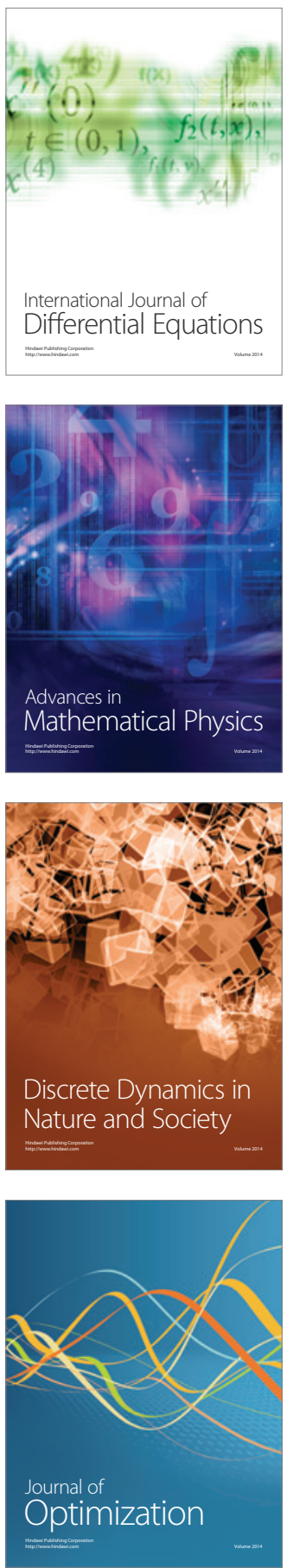